\title{
The role of Piccolo in cancer treatment: relationship with EGFR and related therapies, and a marker for new targeted therapies
}

\author{
Milo Frattini, Francesca Molinari, Samantha Epistolio \\ Laboratory of Molecular Pathology, Institute of Pathology, Locarno, Switzerland \\ Correspondence to: Milo Frattini. Via in Selva, 24, Locarno (6600), Switzerland. Email: milo.frattini@ti.ch. \\ Provenance: This is an invited Editorial commissioned by Section Editor Dr. Hongcheng Zhu (Department of Radiation Oncology, Fudan University \\ Shanghai Cancer Center, Shanghai, China). \\ Comment on: Zhang W, Hong R, Xue L, et al. Piccolo mediates EGFR signaling and acts as a prognostic biomarker in esophageal squamous cell \\ carcinoma. Oncogene 2017;36:3890-902.
}

Submitted Sep 25, 2017. Accepted for publication Oct 05, 2017.

doi: $10.21037 /$ jtd.2017.10.38

View this article at: http://dx.doi.org/10.21037/jtd.2017.10.38

The use of high-throughput technologies becomes more and more relevant every day. Indeed, whole-genome and whole-exome sequencing represent methodologies of particular interest in the discovery of new molecular markers involved in cancer development. In this way, Song and colleagues identified a series of genomic alterations playing a potential relevant role in esophageal squamous cell carcinoma (ESCC) (1). This type of cancer represents the fourth leading cause of cancer-related death (in 2017, the estimated new cases and deaths from ESCC in the USA are 16.940 and 15.690 , respectively) with a very poor 5 -year survival rate (only up to $25 \%$ ) (2). Currently ESCC patients can be managed with various techniques, such as endoscopy, surgery, radiotherapy, and chemotherapy (2). However their effect is limited even if different approaches are combined.

At molecular level, little is known about ESCC, with the consequence that the application of therapies specifically targeting molecular alterations is limited. Therefore, it is of particular relevance to better characterize this type of cancer at molecular level, thus opening the possibility of new therapeutic treatments. One of the new markers identified by Song and colleagues is the PCLO gene, which encodes for a large multi-domain protein, named Piccolo. In the last study of Song and colleagues' group ("Piccolo mediates EGFR signaling and acts as a prognostic biomarker in esophageal squamous cell carcinoma" by Zhang W, Hong R, Xue L, et al., recently published on Oncogene), this protein was extensively characterized, also in terms of interactions with other proteins $(1,3)$.
Piccolo is a presynaptic cytomatrix protein acting as a scaffold in active zone assembly and may modulate synaptic vesicles trafficking and monoamine neurotransmitter release $(4,5)$. Recently, it has been described its involvement in the regulation of specific protein ubiquitination and proteasome-mediated proteolysis (6). Piccolo is characterized by a PDZ domain, $\mathrm{Ca}^{2+} /$ phospholipid binding domains (C2A and C2B) and Zn-finger domains. The PDZ domain is involved in molecular anchoring and assembly at the active zone through the interaction with other presynaptic proteins (7). Furthermore, Piccolo is able to interact with phosphatidylinositol 4,5-bisphosphate (PIP2). All these features suggested that Piccolo may be important in the modulation of membrane trafficking and endocytosis of several membrane receptors, including the epidermal growth factor (EGFR), a member of the ERBB tyrosine kinase receptors family, and one of the most common oncogenes in cancer that is also highly relevant in ESCC cancerogenesis (8).

The PCLO gene is the fifth most frequently mutated gene in ESCC: the Authors found 14 mutations in 13 out of 88 patients (14.7\%), in addition to gene amplification in 16 out of 140 ESCC cases (11.4\%) (3). Using quantitative realtime PCR for the evaluation of PCLO mRNA expression and immunohistochemistry (IHC) for protein expression, Zhang and colleagues found that tumor tissues showed significantly higher levels with respect to matched adjacent normal tissues, thus clearly indicating the involvement of this marker in ESCC development. Furthermore, 
the authors observed a significant association of Piccolo overexpression (at protein level) with the risk of lymph node metastasis, a higher clinical stage, poor overall survival (OS) and poor disease free survival (DFS) $(3,8)$. In vivo experiments based on ESCC cell lines confirmed the association of Piccolo with advanced cases of ESCC, by demonstrating that Piccolo promotes cell proliferation, mobility and enhances the development of ESCC (3). Overall, Piccolo expression can be considered a new prognostic maker for ESCC.

But, most importantly, Zhang and colleagues shed light on the proteins which interact with Piccolo: indeed, based on its features, they hypothesized, and then clearly demonstrated, that Piccolo interacts with EGFR pathway by impairing EGFR internalization: eliminating Piccolo results in decreasing EGFR recycling and influencing the level of EGFR protein. In particular, PCLO silencing increased the ubiquitination level of EGFR. Overall, Piccolo acts via the EGFR pathway to modulate malignancy, in terms of cell growth, colony formation, migration and invasion (2). Moreover they found that ESCC patients with high expression of both Piccolo and EGFR levels had a worse prognosis than patients with high expression of only one of them (3).

Finally, since Piccolo is a membrane protein and may therefore be considered as a potential therapeutic target, Zhang and colleagues generated a murine monoclonal antibody (moAb) against Piccolo and demonstrated that this treatment inhibited cell proliferation in a dose-dependent manner. Moreover, the administration of moAb specifically targeting Piccolo may increase the sensitivity to gefitinib, a tyrosine-kinase inhibitor (TKI) specifically addressed against EGFR. This inhibition repressed in particular the ERK/Akt pathway.

Every study demonstrating the discovery and the involvement of new markers in cancer development (especially ESCC, characterized by a severe course disease) are highly welcome, in particular if it is reported the interaction with known molecular markers for which targeted therapies are available, or if new potentially active targeted drugs can be designed and tested, at least in in vivo experiments. The study of Zhang and colleagues belongs to this category. This study generates a lot of questions, not only concerning Piccolo deregulation itself, but especially about the possibility to explain, and possibly better predict, the efficacy of EGFR targeted therapies in other neoplastic diseases in which EGFR has an important role.

The first case in which the characterization of Piccolo may play a potential role in the identification of patients to be treated with EGFR targeted therapies is head and neck cancer (HNSCC). These tumours involve different area of the head or neck and they usually begin in the squamous cells that line the moist, mucosal surfaces inside the head and neck. In the USA, HNSCC account for approximately $4 \%$ of all cancers and are diagnosed more often among people over 50 years than among younger people (9). Researchers estimated that more than 65,000 people would be diagnosed with HNSCC in 2017 (10). The treatment for HNSCC depends on the location of the tumour and can include surgery, radiation therapy, chemotherapy and targeted therapies. Concerning targeted therapies, at the moment, there are two drugs against EGFR (both moAb) which have received approval from international agencies for the treatment of advanced HNSCC cases: cetuximab and panitumumab. However, there are no markers able to predict the efficacy of these therapies in HNSCC: indeed, neither mutational status nor IHC expression nor gene copy number variations are predictive for EGFR targeted therapies response (11). As a consequence, moAb against EGFR are administered to all metastatic HNSCC patients, although only a minor fraction (about 10\%) may actually benefit from these therapies. The characterization of Piccolo expression (or one of the mechanisms underlining its deregulation, i.e., gene mutations or copy number variations) may identify patients who can really benefit from EGFR targeted therapies and studies investigating this issue are warmly required.

The second example in which Piccolo expression could influence the treatment of patients is colorectal cancer (CRC). CRC is the 3rd most diagnosed cancer in both men and women in Western countries and it is one of the leading causes of cancer related mortality, accounting, worldwide, for more than 600,000 deaths annually (12). Standard of care for patients with CRC is represented by surgery combined with adjuvant chemotherapy or radiotherapy. However, more recently, targeted therapies have been included in the treatment of selected patients with metastatic CRC (mCRC). In particular, several studies have clearly and extensively demonstrated that moAbs targeting EGFR may be effective in the treatment of mCRC patients (13). As for HNSCC, and although some promising preliminary data, it has become clear soon that any method for EGFR evaluation (by assessing protein expression by IHC, gene copy number variations by fluorescent in situ hybridization (FISH) or point mutations by Sanger sequencing) cannot discriminate between patients who are primary resistant 
from those who benefit from EGFR targeted therapies. In particular, it has been shown that there is a discrepancy between EGFR gene status as evaluated by FISH and protein expression assessed by IHC. In this context, by altering the stabilization of EGFR protein, Piccolo may explain these differences and may play a role in the clinical context. On the contrary, a vast consensus has been reached with the identification of some markers that are able to predict the primary resistance to these therapies. For example the occurrence of point mutations in Kirsten rat sarcoma viral oncogene homolog gene (KRAS) and neuroblastoma RAS viral oncogene homolog (NRAS) are molecular alterations constitutively activating the main EGFR downstream pathway (14). However, only a fraction (about $30 \%$ ) of KRAS/NRAS wild-type mCRC patients may really benefit from these therapies (15) and the understanding of the reason why some patients do not respond remains poor. May Piccolo overexpression be a new marker for a better refining of KRAS/NRAS wildtype mCRC patients? Extensive analysis of Piccolo (at protein, mRNA and gene level) in preclinical models and in retrospective and prospective trials is hoped.

On the other hand, the evaluation of Piccolo and the possibility to have moAb against such a molecule may have important influence on the management of mCRC. Since a weak positive correlation between Piccolo and EGFR was observed by Zhang and colleagues, it is conceivable that Piccolo targeted therapies may synergize with and strengthen the efficacy of EGFR-targeted therapies.

Finally, the work of Zhang and colleagues reinforces a new concept in cancer: it seems that it is not only important the deregulation of the marker itself, but also the deregulation of proteins which can interact with the targeted marker. For EGFR, for example, it has been demonstrated the role of NEU3, a plasma membraneassociated sialidase. NEU3 removes sialic acid residues from a variety of glycoconjugates, leading to defects in glycosylation level, a feature known to play a role in cancer malignancy and associated with invasiveness and metastatic potential in cancer cells. Indeed, several studies have shown that NEU3 is up regulated in most cancers and, in particular, in a consistent number of CRC, where it has been demonstrated that NEU3 and EGFR co-immunoprecipitate, thus indicating a clear direct interaction between the two proteins (16). Furthermore, a recent contribution has shown that NEU3 deregulation may enhance EGFR activation without affecting EGFR expression (17). In the paper of Zhang and colleagues, the authors found that Piccolo, by virtue of its ability to influence the ubiquitination level of EGFR, regulates EGFR stability, thus adding a new member to the group of proteins able to interact with EGFR, possibly modulating its activity. New studies evaluating, in the same cohort and in cellular models, the mutual interaction of EGFR, NEU3 and Piccolo are therefore recommended.

In conclusion, as described in the paper by Zhang $\mathrm{W}$, Hong R, Xue L et al., Piccolo protein plays a relevant role in ESCC because it is frequently altered, it is involved in EGFR regulation and it seems to be a prognostic marker for this cancer type $(1,8)$. Researchers are now faced on the need to investigate also the deregulation of protein potentially interacting with the marker under investigation. Data coming from these analyses may lead to a substantial revolution in the evaluation of predictive biomarkers, in particular, new studies investigating in more details the interaction between EGFR and Piccolo, and the clinical consequences of this interplay, must be performed in the near future.

Furthermore, the possibility to produce moAb against Piccolo may open the way to new treatments for patients affected by Piccolo and by EGFR deregulation.

\section{Acknowledgements}

None.

\section{Footnote}

Conflicts of Interest: The authors have no conflicts of interest to declare.

\section{References}

1. Song Y, Li L, Ou Y, et al. Identification of genomic alterations in oesophageal squamous cell cancer. Nature 2014;509:91-5.

2. Higuchi K, Koizumi W, Tanabe S, et al. Current management of esophageal squamous-cell carcinoma in Japan and other countries. Gastrointest Cancer Res 2009;3:153-61.

3. Zhang W, Hong R, Xue L, et al. Piccolo mediates EGFR signaling and acts as a prognostic biomarker in esophageal squamous cell carcinoma. Oncogene 2017;36:3890-902.

4. Zhai RG, Vardinon-Friedman H, Cases-Langhoff C, et al. Assembling the presynaptic active zone: a characterization of an active one precursor vesicle. Neuron 2001;29:131-43. 
5. Fenster SD, Kessels MM, Qualmann B, et al. Interactions between piccolo and the actin/dynamin-binding protein Abp1 link vesicles endocytosis to presynaptic active zones. J Biol Chem 2003;278:20268-77.

6. Ivanova D, Dirks A, Fejtova A, et al. Bassoon and piccolo regulate ubiquitination and link presynaptic molecular dynamics with activity-regulated gene expression. J Physiol 2016;594:5441-8.

7. Fenster SD, Chung WJ, Zhai R, et al. Piccolo a presynaptic zinc finger protein structurally related to bassoon. Neuron 2000;25:203-14.

8. Shang L, Liu HJ, Hao JJ, et al. A panel of overexpressed proteins for prognosis in esophageal squamous cell carcinomas. PLoS One 2014;9:e111045.

9. Siegel RL, Miller KD, Jemal A. Cancer statistics 2017. CA Cancer J Clin 2017;67:7-30.

10. American Cancer Society: cancer facts \& figures 2017. American Cancer Society, 2017. Available online: https:// www.cancer.org/research/cancer-facts-statistics/all-cancerfacts-figures/cancer-facts-figures-2017.html

11. Siano M, Molinari F, Martin V, et al. Multicenter phase II study of panitumumab in platinum pretreated, advanced head and neck squamous cell cancer. Oncologist 2017;22:782-e70.

Cite this article as: Frattini M, Molinari F, Epistolio S. The role of Piccolo in cancer treatment: relationship with EGFR and related therapies, and a marker for new targeted therapies. J Thorac Dis 2017;9(11):4240-4243. doi: 10.21037/ jtd.2017.10.38
12. Ferlay J, Parkin DM, Steliarova-Foucher E. Estimates of cancer incidence and mortality in Europe in 2008. Eur J Cancer 2010;46:765-81.

13. Dienstmann R, Tabernero J. Spectrum of gene mutations in colorectal cancer. Cancer J 2016;22:149-55.

14. Loupakis F, Pollina L, Stasi I, et al. PTEN expression and KRAS mutations on primary tumors and metastases in the prediction of benefit from cetuximab plus irinotecan for patients with metastatic colorectal cancer. J Clin Oncol 2009;27:2622-9.

15. Linardou H, Dahabreh IJ, Kanaloupiti D, et al. Assessment of somatic k-RAS mutations as a mechanism associated with resistance to EGFR targeted agents: a systematic review and meta-analysis of studies in advanced non-smallcell lung cancer and metastatic colorectal cancer. Lancet Oncol 2008;9:962-72.

16. Miyagi T, Takahashi K, Moriya S, et al. Altered expression of sialidases in human cancer. Adv Exp Med Biol 2012;749:257-67.

17. Mozzi A, Forcella M, Riva A, et al. NEU3 activity enhances EGFR activation without affecting EGFR expression and acts on its sialylation levels. Glycobiology 2015;25:855-68. 